Quantum Monte Carlo Assessment of the Relevance of Electronic Correlations in Defects and EOS in Metals

R. Q. Hood, A. J. Williamson, J. L. Dubois, F. A. Reboredo

February 11, 2008 
This document was prepared as an account of work sponsored by an agency of the United States government. Neither the United States government nor Lawrence Livermore National Security, LLC, nor any of their employees makes any warranty, expressed or implied, or assumes any legal liability or responsibility for the accuracy, completeness, or usefulness of any information, apparatus, product, or process disclosed, or represents that its use would not infringe privately owned rights. Reference herein to any specific commercial product, process, or service by trade name, trademark, manufacturer, or otherwise does not necessarily constitute or imply its endorsement, recommendation, or favoring by the United States government or Lawrence Livermore National Security, LLC. The views and opinions of authors expressed herein do not necessarily state or reflect those of the United States government or Lawrence Livermore National Security, LLC, and shall not be used for advertising or product endorsement purposes.

This work performed under the auspices of the U.S. Department of Energy by Lawrence Livermore National Laboratory under Contract DE-AC52-07NA27344. 


\title{
Quantum Monte Carlo Assessment of the Relevance of Electronic Correlations in Defects and EOS in Metals
}

\author{
R. Q. Hood, A. J. Williamson, J. L. Dubois, and F. A. Reboredo
}

\begin{abstract}
We have developed a highly accurate computational capability to calculate the equation of state (EOS) and defect formation energies of metallic systems. We are using a newly developed algorithm that enables the study of metallic systems with quantum Monte Carlo (QMC) methods. To date, technical limitations have restricted the application of QMC methods to semiconductors, insulators and the homogeneous electron gas. Using this new "QMC for metals" we can determine, for the first time, the significance of correlation effects in the EOS and in the formation energies of point defects, impurities, surfaces and interfaces in metallic systems. These calculations go beyond the state-of-the-art accuracy which is currently obtained with Density Functional Theory approaches. Such benchmark calculations can provide more accurate predictions for the EOS and the formation energies of vacancies and interstitials in simple metals. These are important parameters in determining the mechanical properties as well as the micro-structural evolution of metals in irradiated materials or under extreme conditions. We describe the development of our "QMC for metals" code, which has been adapted to run efficiently on a variety of computer architectures including BG/L. We present results of the first accurate quantum Monte Carlo calculation of an EOS of a realistic metallic system that goes beyond the homogeneous electron gas.
\end{abstract}




\section{INTRODUCTION/BACKGROUND}

One of the challenges for computational models used to predict material failure is to understand changes in a material's mechanical properties caused by aging in a harsh environment. In most metals, the mechanical properties are dominated by the formation energies and migration energy barriers of defects. These defects can be generated by alloying, radiation damage, plastic deformation, or thermal processing. When a material is in thermal equilibrium, the abundance of a given defect is directly related to its formation energy. Outside thermal equilibrium, the situation is more complex: a combination of the rate of defect diffusion (determined by the migration energy barrier), the stress field around a defect (related to its formation volume) and the binding energy of the various defect clusters decides the micro-structural evolution. Therefore, to predict, for example, the rate of void growth and swelling in an irradiated metal, one must be able to accurately calculate the formation energies and mobilities of defects.

From a theoretical point of view, the formation energies of most defects in a crystal can be decomposed into a long range elastic energy and a core energy. The long range energy can be obtained from continuum elasticity theory. As one approaches the core, atomistic calculations are required for two main reasons; (i) the atomic displacements are large and inter-atomic distances differ substantially from the bulk so elasticity theory fails, and (ii) the chemical bonding and atomic coordination are different.

To date, atomistic calculations of metals have been restricted to empirical interatomic potentials, (e.g. the Embedded Atom Method [1]), empirical tight binding models [2], quantum based interatomic potentials, [3] and first principles Density Functional Theory (DFT) calculations. $[4,5]$ The empirical models contain adjustable parameters that are fitted to experimental data and ab initio results. Only DFT calculations incorporate quantum mechanical effects without adjustable parameters.

DFT has proven to be an accurate tool for calculating a wide range of bulk and surface properties of materials. It has demonstrated excellent agreement with experiments for a range of properties such as lattice parameters and elastic constants. However, there are also well known errors of DFT such as the "band gap error" band gaps are underestimated by $50 \%$ or more, and the "LDA over-binding error" in which LDA functionals typically predict bond lengths that are shorter than measured values. In some cases, the origin of DFT errors 
has been traced to the mean field treatment of electron correlations. More accurate values have been calculated by including dynamical electron correlations with configuration interaction (CI) or Quantum Monte Carlo (QMC) methods. [6] For example, diffusion quantum Monte Carlo calculations of the formation energies of point defects and surface energies in semiconductors have shown that important corrections arise when quantum correlations are fully taken into account. [7] Additionally, activation energies of common chemical reactions obtained by DFT methods have been shown to differ substantially from benchmark QMC values. [8]

For metallic systems, the size of the errors in DFT calculations is largely unknown, as more accurate QMC benchmark calculations do not currently exist. However, recent variational Monte Carlo calculations have demonstrated that important differences are expected for highly inhomogeneous electron gases. [9] Under extreme temperatures and pressures, when accurate experimental measurements become increasingly difficult, first principles DFT calculations currently provide the only source of data for fitting empirical interatomic potentials. Therefore, any inaccuracies related to the mean-field correlations used in DFT will propagate to the predictions made by the empirical codes fit to DFT. In this project, we are quantitatively evaluating the errors in DFT calculations of metals for the first time.

There are contrary points of view on the errors in DFT for metals which make evident the need for accurate QMC calculations. As correlations are affected by the value of the gap, there is good reason to expect that correlation effects will be more important in systems without an energy gap, i.e., metals. On the other hand, in cases where the electronic density is nearly homogeneous, DFT methods are expected to be most accurate as the correlation effects included in DFT functionals are derived from QMC calculations for the homogeneous electron gas. Similarly, one might expect that correlation errors in DFT calculations of defects such as stacking faults, which do not induce strong changes in the density, would be less significant than in calculations of vacancies, surfaces, and dislocations, where the density changes significantly. This could, in turn, lead to errors in the relative formation energies of dislocations, staking faults, and partial dislocations which are used to determine the relative abundance of these defects (i.e., a single dislocation vs. an extended dislocation with two partials and a staking fault) which, in turn, determine the mechanical properties of the material. Therefore, a quantitative evaluation of the errors of DFT is required for a better understanding of the properties of these materials across all length scales. 
While it would clearly be valuable to perform benchmark QMC calculations for metals, QMC calculations for metals have historically lagged behind similar calculations for semiconductors. The reason is that, for metals, one must carefully evaluate the structure of the Fermi surface. This requires the calculation of a larger number of electronic energy levels. For QMC calculations this corresponds to calculating a large number of electrons.

\section{RESEARCH ACTIVITIES}

Quantum Monte Carlo (QMC) methods offer a direct and accurate wave function-based treatment of quantum many-body effects. The trial wavefunction is central to QMC, as it controls both the statistical variance and the final accuracy obtained. For the many-electron trial wavefunction we use the standard Slater-Jastrow form

$$
\Psi(\mathbf{R})=e^{J(\mathbf{R})} \sum_{i} a_{i} D_{\uparrow i}\left(\mathbf{r}_{1}, \mathbf{r}_{2}, \ldots, \mathbf{r}_{N_{\uparrow}}\right) D_{\downarrow i}\left(\mathbf{r}_{N_{\uparrow}+1}, \ldots, \mathbf{r}_{N}\right)
$$

where the configuration $\mathbf{R}=\left(\mathbf{r}_{1}, \mathbf{r}_{2}, \ldots, \mathbf{r}_{N}\right)$ is made up of the coordinates of the spin-up and spin-down electrons with $N$ being the total number of electrons. The Jastrow correlation factor is $e^{J(\mathbf{R})}$ in which $J$ consists of one-, two-, and three-body terms. The spin-up and spin-down determinants $D_{\uparrow i}$ and $D_{\downarrow i}$, respectively are formed from single-particle orbitals obtained from density functional theory calculations. It is the $N^{3}$ scaling of the calculation of the orbitals in the determinants which dominates the cost of a QMC simulation. These orbitals however can be localized in real space by transforming them into an orthogonal Wannier basis. [10] Truncating the Wannier orbitals reduces the number of evaluations for a configuration $\mathbf{R}$ and results in a linear scaling in electron number. This approach has been successfully applied to the study of many semiconductor systems, evaluating, for the first time, non-trivial surface formation energies. [11] However, until recently, we have not been able to implement this linear scaling method in metallic systems. This is because Wannier orbitals decay exponentially in semiconductors and insulators but only decay polynomially in metals. We have developed a novel non-orthogonal transformation [12] that overcomes this limitation and enables us to perform QMC calculations for metals.

Generating optimal nonorthogonal orbitals in a metallic system is more challenging algorithmically than constructing orthogonal Wannier orbitals in semiconductors. One must insure that the transformation matrix that connects the original density functional single- 
particle orthogonal orbitals with the nonorthogonal orbitals is not singular. Our approach is based on algorithms developed for linear scaling DFT calculations [13-18] and is designed to minimize a cost function associated with the total number of orbital evaluations required in a linear scaling QMC calculation. We translated this algorithm into an efficient parallel code that allowed us to generate optimal nonorthogonal orbitals in real non-homogeneous metals. Applied to metallic aluminum, using a sufficiently large number of points in momentum space (k-points) to accurately represent the Fermi surface, we were able to localize and truncate nonorthogonal orbitals. We integrated this code into our standard density functional electronic-structure software packages and QMC code CASINO. [19]

The evaluation of the orbitals, even if they are localized and truncated, takes the majority of the computation time. Thus it is essential to develop representations of the orbitals that can be evaluated efficiently. Because we require the orbitals at points in real space, the most natural procedure is to use a basis localized in real space. This way the cost of evaluation of the orbitals does not grow with system size, as would be the case if the orbitals are represented in terms of plane waves. The density functional codes we use output single particle orbitals expanded in plane waves. A portion of our orbital localization code converts the plane wave representation of each orbital to a grid representation using cubic splines, know as blips. [20] Our QMC calculations of metals are over a thousand times faster when we use a blip rather than a plane-wave representation.

The downside of using a blip basis is the large amount of computer memory required, of the order of ten gigabytes for systems with around a thousand electrons. In the standard version of CASINO each processor stores all of the orbitals since it must independently evaluate the trial wavefunction for its own walk in configuration space. We generalized the CASINO code such that each processor only needs to store and evaluate a portion of the orbitals. This introduced some additional overhead, of around twenty percent, involving the communication and synchronization of the orbital evaluations. With this change the size of our calculations are no longer memory bound. We can perform QMC simulations on any computer architecture including memory limited systems like BG/L. The formatted files that store the blip represented orbitals between different QMC calculations have been as large as sixty gigabytes. Reading such a file and distributing the data to the other processors at the beginning of a QMC calculation can take as long as an hour. For this reason we added a feature to CASINO that allows one to store the orbitals as unformatted files, and made a 
more judicious use of MPI routines for communicating the data.

In a QMC calculation independent walkers wander through the $3 \mathrm{~N}$ dimension configuration space with each electron moving in turn. Updating the determinants in the trial wavefunction after a single electron move requires evaluating all of the single-particle orbitals at the same electron position. Existing blip evaluators did not take full advantage of the fact that several operations can be eliminated when calculating the values of several different orbitals at the same coordinates. We wrote a blip evaluator from scratch that was over ten times faster than the best one previously available. This alone translated into an overall factor of five speedup in our QMC calculations of metals.

Using a finite simulation cell with periodic boundary conditions in QMC calculations to model an extended system like a metal introduces finite-size effects. One source of finite-size effects is the electronic Coulomb interaction, that is often treated using an Ewald interaction. We instead use a model periodic Coulomb interaction [21] that has been shown to dramatically reduce the finite-size effects in the interaction energy. In large systems the evaluation of this model periodic Coulomb interaction as originally implemented in CASINO can take a majority of the computers resources. By representing a portion of this interaction using blips we obtained an additional three fold reduction in overall computer time.

In QMC calculations of solids the Slater determinants in the trial wavefunctions usually are made up of the DFT orbitals that have the lowest single-particle DFT energies. The presence of a Fermi surface in a metal complicates this approach. In DFT calculations the single-particle states at the Fermi energy are partially occupied to insure that the electronic density has the correct symmetry. A DFT calculation of a metal utilizing partial occupations formally goes beyond a wavefunction based approach relying instead upon a statistical density-matrix formalism. In QMC a determinant in the Slater-Jastrow trial wavefunction either contains a DFT orbital or it doesn't. Partial occupations are not possible. A finite sum of the determinants composed of DFT orbitals will not in general produce an electronic density with the correct symmetry. Trial wavefunctions constructed using different occupations of the single-particle states at the Fermi energy will in general produce different QMC energies. Our QMC tests in a homogeneous metal revealed that the energy differences between different fillings of the states were less than $0.02 \mathrm{eV} /$ atom.

The first application of our "QMC for metals" code was to aluminum. We used a simulation cell with 256 aluminum atoms, containing 768 valence electrons, which we found to 
be large enough to insure that finite-size effects were negligible. Shown in Fig. 1 are plots of the QMC energy as a function of the lattice constant $a$ for FCC aluminum. Our first QMC calculations (green circles) were with trial wavefunctions, Eq. 1, that consisted of one up-spin and one down-spin determinant. The unphysical discontinuity at around $a=3.98 \AA$ originated from a band crossing at the Fermi energy. At each value of $a$ we fill the determinants using the lowest energy single-particle orbitals taken from a DFT calculation. At around $a=3.98 \AA$ the symmetry of the highest energy orbital contained in the determinants changes. This change in symmetry changes the symmetry of the many-electron nodal surface of the trial wavefunction. This effects the total QMC energy since we use the fixednode approximation; the standard approach used in all large-scale QMC applications. This type of discontinuity is unique to metals and cannot arise in fixed-node QMC calculations of semiconductors $[22,23]$ and insulators [24] because of their electronic gaps.

With the aim of removing the unphysical discontinuity in Fig. 1 we used trial wavefunctions with six up-spin and six-down spin determinants. The determinants were filled with orbitals that were either occupied in the DFT calculation or had DFT single-particle energies slightly higher than the Fermi energy. We first optimized the coefficients in $a_{i}$ in Eq. 1 using variance minimization, $[25,26]$ shown as red circles in Fig. 1. The calculated QMC energy was a physically smooth function of $a$. At some values of $a$ the QMC energies with six up-spin and down-spin determinants were higher than with one up-spin and down-spin determinant. The six determinant trial wavefunctions had been constructed, such that when the values of $a_{i}$ in Eq. 1 took certain combinations of 1 and 0, one would obtain the one determinant trial wavefunction, shown as green circles in Fig. 1. Thus the six determinant trial wavefunctions had a larger variational freedom than the one determinant trial wavefunction. This larger variational freedom, however, did not always result in a lower energy. While it was known that minimizing the variance of the trial wavefunction is no guarantee that the corresponding fixed-node QMC energy would be lowered as the variational freedom is enhanced, there had been no known examples until now of this possibility. Recently an effective method [27-29] has been developed for optimizing a trial wavefunction by minimizing the energy that is applicable to large systems. During this project this method was implemented and tested within our "QMC of metals" code. Optimizing the coefficients $a_{i}$ in Eq. 1 of the same six determinant trial wavefunctions using energy minimization we obtained the results shown as black cirles in Fig. 1. To within the statistical error bars this 
approach gave the lowest energies and produced results that were physically smooth. This demonstrated that energy minimization of multiple-determinant trial wavefunctions is an effective approach to calculating the properties of metals. The solid black line in Fig. 1 is a quartic fit to the black circles. This gives a QMC calculated ground-state equilibrium lattice constant of $3.94 \AA$. After removal of the finite-temperature and zero-point effects the experimental equilibrium lattice constant [30] is $4.02 \AA$. For comparison the DFT (local density approximation) calculated ground-state equilibrium lattice constant is $3.96 \AA$. Subsequent calculations suggest that the deviation of our calculated equilibrium lattice constant from experiment originates mostly from using a DFT-based pseudopotential rather than from the fixed-node error. We are actively investigating this issue by carrying out calculations using a different DFT-based pseudopotential and using trial wavefunctions with very different nodal surfaces.

\section{EXIT PLAN}

The development of an accurate approach for calculating the equation of state of metals from first-principles is important for the ASC program. We are currently using funds from this program to continue our development of a "QMC for metals" code and to begin calculations of beryllium, a programmatically relevant metal. Our development of the computational and the algorithmic capabilities in this area has given us the tools to conduct highly visible research that cannot be performed by other groups in the world. The next phase will involve calculating the most accurate properties of defects in metals to date. Research in this area was slowed by the departure of the two previous principal investigators, Reboredo and Williamson, from the laboratory. This project could evolve into the study of metallic systems with increasing complexity relevant for programmatic applications. The results obtained can be used to benchmark DFT calculations and to fit quasi-classical potentials for large scale simulations that are currently used to describe matter at extreme conditions. Additionally, QMC calculations of realistic metallic systems could help the development of better density functionals by providing data outside the constant density regime.

This work performed under the auspices of the U.S. Department of Energy by Lawrence Livermore National Laboratory under Contract DE-AC52-07NA27344. 


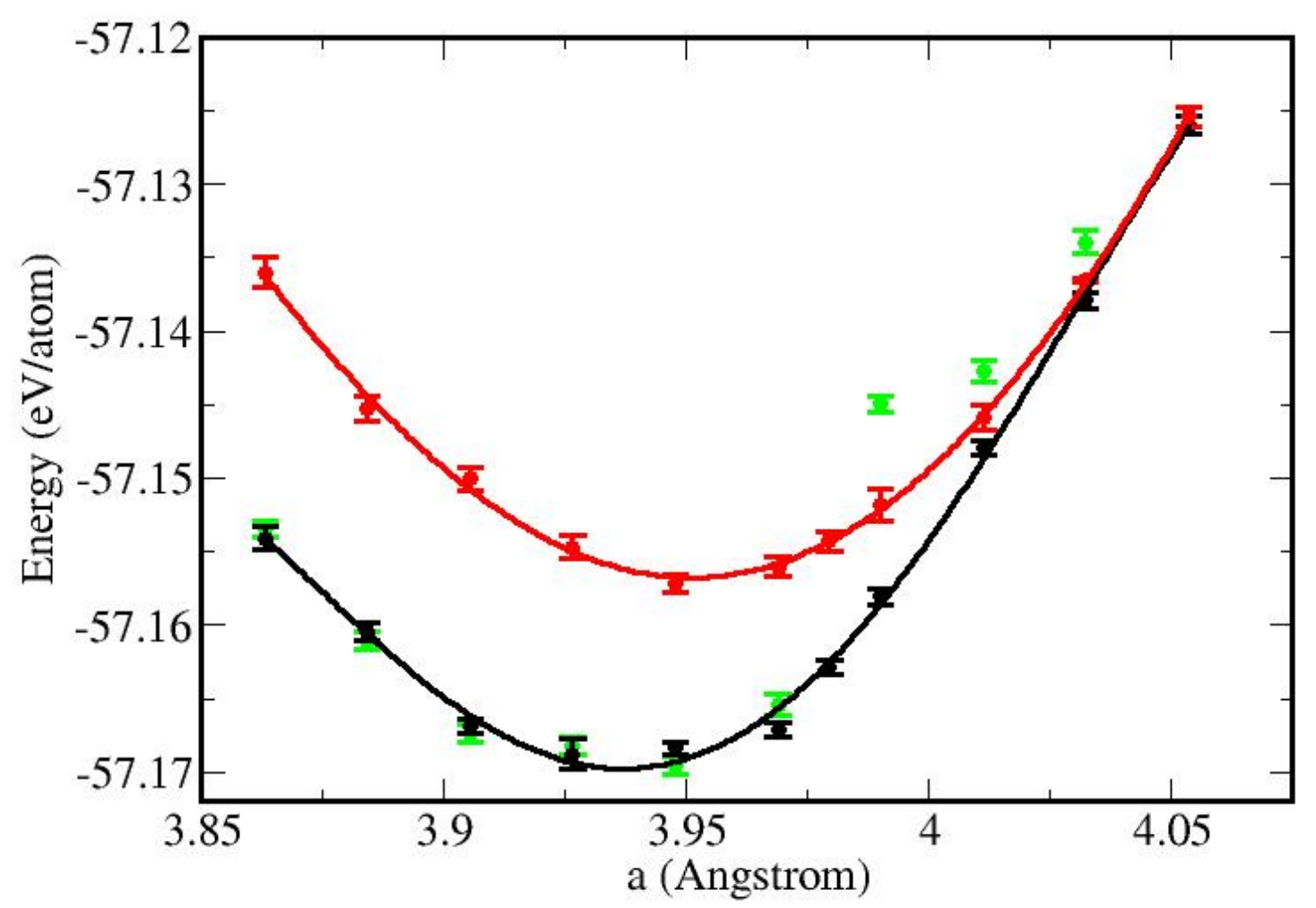

FIG. 1: QMC energies (eV/atom) of FCC aluminum as a function of the lattice constant $a(\AA)$ calculated using a Slater-Jastrow trial wavefunction consisting of one up-spin and one down-spin determinant (green circles) and six up-spin and six down-spin determinants in which the coefficients $a_{i}$ in Eq. 1 were optimized using variance minimization (red circles) and energy minimization (black circles). The statistical error bars are shown. The red and black solid lines are quartic fits (energy as a function of $a$ ) corresponding to the red and black circles, respectively. 
[1] S. J. Zhou, D. L. Preston, P. S. Lomdahl, and D. M. Beazley, Science 279, 1525 (1998).

[2] M. J. Mehl, D. A. Papaconstantopoulos, N. Kioussis, and M. Herbranson, Phys. Rev. B 61, $4894(2000)$.

[3] J. A. Moriarty, J. F. Belak, R. E. Rudd, P. Soderlind, F. H. Streitz, and L. H. Yang, J. Phys.: Condens. Matter 14, 2825 (2002).

[4] D. Roundy, C. R. Krenn, M. L. Cohen, and J. W. Morris, Phys. Rev. Lett. 82, 2713 (1999).

[5] G. Liu, N. Kioussis, V. V. Bulatov, and F. Kaziras, Phys. Rev. B 62, 3099 (2000).

[6] For a review of QMC see W.M.C. Foulkes, L. Mitas, R.J. Needs, and G. Rajagopal, Rev. Mod. Phys. 73, 33 (2001).

[7] W. -K. Leung, R. J. Needs, G. Rajagopal, S. Itoh, and S. Ihara, Phys. Rev. Lett. 83, 2351 (1999).

[8] J. C. Grossman, L. Mitas, Phys. Rev. Lett. 79, 4353 (1997).

[9] M. Nekovee, W. M. C. Foulkes, R. J. Needs, Phys. Rev. Lett, 87, 036401 (2001).

[10] A. J. Williamson, R. Q. Hood, and J. C. Grossman, Phys. Rev. Lett. 87, 246406 (2001).

[11] A. Puzder, A. J. Williamson, F. A. Reboredo, G. Galli, Phys. Rev. Lett. 91, 157405 (2003).

[12] F. A. Reboredo, A. J. Williamson, Phys. Rev. B 71, 121105 (2005).

[13] G. Galli, Phys. Status Solidi B 217, 231 (2000).

[14] X. Li, R. Nunes, and D. Vanderbilt, Phys. Rev. B 47, 10891 (1993).

[15] F. Mauri and G. Galli, Phys. Rev. B 50, 4316 (1994).

[16] J. Kim, F. Mauri, and G. Galli, Phys Rev. B 52, 1640 (1995).

[17] E. Hernandez and M. Gillan, Phys. Rev. B 51, 10157 (1995).

[18] S. Liu, J. Perez-Jorda, and W. Yang, J. Chem. Phys. 113, 1634 (2000).

[19] R. J. Needs, M. D. Towler, N. D. Drummond and P. Lopez Rios, CASINO code, Version 2.1 User's Manual, (University of Cambridge, Cambridge 2007).

[20] D. Alfe and M. J. Gillan, Phys. Rev. B 70, 161101 (2004).

[21] A. J. Williamson, G. Rajagopal, R. J. Needs, L. M. Fraser, W. M. C. Foulkes, Y. Wang, and M. -Y. Chou, Phys. Rev. B 55, R4851 (1997).

[22] A. Maezono, A. Ma, M. D. Towler, and R. J. Needs, Phys. Rev. Lett. 98, 025701 (2007).

[23] D. Alfe, M. J. Gillan, M. D. Towler, and R. J. Needs, Phys. Rev. B 70, 214102 (2004). 
[24] N. D. Drummond and R. J. Needs, Phys. Rev. B 73, 024107 (2006).

[25] C. J. Umrigar, K. G. Wilson, and J. W. Wilkins, Phys. Rev. Lett. 60, 1719 (1988).

[26] N. D. Drummond and R. J. Needs, Phys. Rev. B, 72, 1 (2005).

[27] M. P. Nightingale and V. Melik-Alaverdian, Phys. Rev. Lett. 87, 043401 (2001).

[28] C. J. Umrigar, J. Toulouse, C. Filippi, S. Sorella, and R. G. Henning, Phys. Rev. Lett. 98, $110201(2007)$.

[29] J. Toulouse and C. J. Umrigar, J. Chem. Phys. 126, 084102 (2007).

[30] R. Guadoin and W. M. C. Foulkes, Phys. Rev. B 66, 052104 (2002). 